Proceedings

\title{
Demand Response: Moving beyond the Technical and Physical Context of Buildings ${ }^{\dagger}$
}

\author{
Sylvia Breukers ${ }^{1, *}$ and Tracey Crosbie ${ }^{2}$ \\ 1 DuneWorks, Eschweilerhof 56, 5625 NN Eindhoven, The Netherlands \\ 2 Technology Futures research institute, Campus Heart, Teesside University, Middlesbrough TS1 3BX, UK \\ * Correspondence: sylvia.breukers@duneworks.nl \\ † Presented at the Sustainable Places 2019 (SP 2019), Sardinia, Italy, 5-7 June 2019.
}

Published: 12 August 2019

\begin{abstract}
This paper discussed the demonstration experiences from an EU funded H2020 project called "Demand Response in Blocks of Buildings" (DR-BOB). The project developed a Demand Response solution and demonstrated that solution at four blocks-of-buildings sites. Based on a comparative analysis of qualitative results from four pilot sites at which the DR BOB solution was demonstrated, we present key lessons that are relevant to consider when initiating demand response at the level of blocks-of-buildings. What has become clear is that organisational (including user-related) conditions are highly relevant-but understudied-for successful DR at a BOB. In order to reflect this finding, we present a framework to assess three key readiness levels of blocksof-buildings (BOB) for demand response (DR) in which organisational readiness is included-next to physical, technological and market readiness.
\end{abstract}

Keywords: demand response; organisational readiness; sociotechnical innovations; users; end-users

\section{Introduction: The Challenges of Medium-Scale Demand Response}

From January 2018 until January 2019, demonstrations of Demand Response solutions for Blocks-of-Buildings (BOBs) were conducted at four different sites in Italy, UK, Romania and France. As part of the EU funded H2020 project called "Demand Response in Blocks of Buildings" (DR-BOB), an evaluation of these demonstrations has been performed. And based on a comparative analysis of the qualitative evaluation results from these four pilot sites at which the DR BOB solution was demonstrated, this paper presents key findings, recommendations and conclusions.

\subsection{Demand Response for Blocks-of-Buildings: What Is New?}

Large-scale industrial Demand Response involves changes in energy consumption patterns and volumes that are rather straightforward and relatively easy to control and assess because of the participation of few large assets - which often can be controlled using remote and/or automated control mechanisms. As for medium-scale DR however, the situation is quite different. Based on the experiences of the DR BOB project, a picture emerges of Blocks-of-Building sites with multiple dispersed buildings of diverse ages and with very different building characteristics and-qualities. In addition, diversity is also apparent in the sort of assets, the activities and processes, the building users and their schedules and routines. This paper addresses the challenges faced in dealing with the disperse and multifaced nature of BOBs when planning for DR interventions.

\subsection{The 4 Demonstration Sites of the DR BOB Project}

The DR-BOB solution has been piloted at four demonstration sites: two university campuses (one in Middlesbrough in the UK and one in Cluj Napoca in Romania), a business park in Anglet 
(Technology campus Anglet) in France, and a private hospital in Brescia in Italy. With local variations in the exact organisation of the elements, the technical part includes the following elements [1]:

- $\quad$ A Demand Response Manager (DRM) provided by Siemens DEMS ${ }^{\circledR}$, providing macro-level optimized energy management for aggregators that seek to optimize energy management for DR in multiple blocks of buildings.

- A Local Energy Manager (LEM) provided by Teesside University, for micro level optimised energy management for DR at the level of a single block of buildings.

- A Consumer Portal provided by GridPocket's EcoTroks ${ }^{\mathrm{TM}}$, providing user interfaces for facilities managers and for building occupants at each block of buildings.

\section{Materials and Methods: A Qualitative Analysis of Process of the Demonstration of the DR BOB Solution at Four Sites}

Our data included interviews, informal talks and an extensive questionnaire taken before, during and after the demonstrations with the 4 partners that coordinated the demonstrations at each site. The findings were analysed with attention to the preparation of the demonstration, the implementation of the demonstration and the evaluation of the demonstration. Challenges that were reported on were structured into physical and technological aspects; market-related conditions; and organizational (and user-related) conditions. Table 1 summarizes these as so-called readiness levels (more elaboration on these in [2]). Based on the main findings for each demo-site a comparative analysis was performed.

Table 1. Readiness levels.

\begin{tabular}{|c|c|}
\hline $\begin{array}{c}\text { Physical and tech- } \\
\text { nological readi- } \\
\text { ness: }\end{array}$ & $\begin{array}{l}\text { The buildings, the technologies deployed in the buildings and their building management systems, } \\
\text { controllable assets and energy metering. The characteristics of the buildings and their technologies, } \\
\text { systems and assets affect: } \\
\text { - the implementation of the DR solutions and the running of the DR events. } \\
\text { - the fit with DR BOB software and hardware and what needs to be adapted and/or changed and } \\
\text { how }\end{array}$ \\
\hline Market readiness: & $\begin{array}{l}\text { The maturity of national DR markets and regulations varies significantly in different EU countries. } \\
\text { This impacts on; } \\
\text { - The possibility for different stakeholders in the value chain for DR to realize economic and/or } \\
\text { other benefits from implicit and explicit DR in BOBs, } \\
\text { - How the DR BOB solution allocates value to increase the attractiveness of the solution for market } \\
\text { actors and potential customers, which has distributive consequences related to which stakehold- } \\
\text { ers benefit from DR }\end{array}$ \\
\hline $\begin{array}{l}\text { Organisational } \\
\text { readiness: }\end{array}$ & $\begin{array}{l}\text { Organisational culture and dynamics, engagement and commitment, level of being informed or en- } \\
\text { ergy aware and mind-set of building management and building users. This impacts on: } \\
\text { - The extent to and ways in which DR fits and the successful deployment of DR. } \\
\text { - The extent to which direct users (BM/FM/EM) and indirect building users (building occupants) } \\
\text { are likely to respond positively to DR. }\end{array}$ \\
\hline
\end{tabular}

\section{Results: Readiness Levels at the Demo-Sites}

Table 2 shows that at both the English and French sites eventually the physical and technological challenges have been solved, while at the Italian and Romanian site some challenges have remained/have not all been solved. As for organizational readiness, that has been challenging at all the sites in different manners. And for market readiness, only in the UK there is a fully developed market for DR. 
Table 2. Comparison of readiness levels at the 4 demo-sites.

\begin{tabular}{cccc}
\hline & Physical and Technological Readiness & Organisational Readiness & Market Readiness \\
\hline English Pilot & + & $+/-$ & + \\
French pilot & + & $+/-$ & $+/-$ \\
Romanian pilot & +- & $+/-$ & - \\
Italian pilot & $+/-$ & $+/-$ & - \\
\hline
\end{tabular}

BM: Building Managers; FM: Facility Managers; EM: Energy Managers. We use BM/FM/EM to denote all or any of these actors, considering that their role in relation to the buildings and in relation to DR in the $\mathrm{BOB}$ is to some extent context dependent.

In terms of physical and technological readiness, it has become clear that not only the age of a building, but also its function affects how well DR can be implemented. University campuses as well as hospital areas often consist of a combination of old historic buildings and (sometimes very new and smart) buildings. TUCN in Romania provides an example of how the monumental status of a building can hinder possibilities for retrofitting. In such a situation, DR could be a way to contribute to overall $\mathrm{BOB}$ energy optimization. If the retrofitting of the older buildings is not a problem, then DR may not be a first logical step from the perspective of the building owner who might opt for energy efficiency measures first. Very new buildings can make DR easier to implement, in as far as the DR technologies are likely to be more compatible with new building installations compared to older installations in old buildings. Generally, older machines are not made for DR and they are not all equally suitable to participate. However, even in very new and smart buildings-e.g., in the case of the hospital in Brescia where energy-intensive critical medical interventions like surgery are performed in the smartest buildings - other considerations prevent opportunities for DR, when it is not allowed for safety reasons (or if it's not (yet) economically feasible for these activities). Of course, the fact that these rules applied in the Italian hospital case does not mean that these rules apply similarly in other hospitals, but it is something to investigate when developing DR propositions specifically for hospital sites. The French case revealed how a set of buildings that is not yet physically or ICT connected can bring additional challenges, when these connections have to be physically developed, in agreement with (IT) management at all the buildings. Another issue that emerged is that different organisations will impose limits on the extent to which company-specific data are to be shared. At all sites there were challenges related in matching the DR BOB solution with the existing technological systems and in some cases additional costs were necessary to solve these. The French and UK sites have managed to solve these issues best, although it did result in project demonstration delays.

In terms of market readiness, the current situation and expectations with regard to future opportunities diverge. Only in the UK is explicit DR an option currently. However, at all demo-sites there is the expectation that both implicit and explicit DR will become an (easier) option. In Romania, main concerns are a lack of knowledge and competence that are needed to design and implement the necessary regulation, but there is little doubt that it will happen (the more since the current balancing approach is unsustainable both economically and ecologically). In Italy, one challenge that becomes clear from an experimental explicit DR programme relates to the fact that participating BOBs must guarantee a high minimum amount of flexibility loads which was not an option for the hospital in Brescia-due to the safety considerations. The French market offers opportunities for collecting the flexibility of several BOBs, and the same is already the case for the UK. And what became particularly clear from the Italian case is that as long as the value generated with participation in DR events is not tangible, responsiveness will be limited.

In terms of organisational readiness and user-related issues, several issues stand out. Organisational commitment differs at the different sites, but at all sites it became clear that organisational (energy-related) awareness is an essential condition to make DR work at a BOB for several reasons. Without organisational awareness and engagement, it is unlikely that current practices in scheduling, which at Teesside are highly inflexible and therewith also limit possibilities for participation in DR events, will change. Organisational commitment is also important to ensure that efforts done by the $\mathrm{FM} / \mathrm{EM} / \mathrm{BM}$ who are crucial to make DR work are seen and appreciated- 
e.g., by awarding them resources like extra time. But organisational commitment at the top and middle levels is also important as it gives expression to a culture and social norm towards building occupants who then may feel more engaged as well.

A major restriction on DR came from scheduling and operational restrictions-most clearly visible at the UK and Italian demo-sites. The timing of events generally was an issue at all the demosites, as well as the timing of the notifications about the events. Especially when active engagement of participants was asked for, timing of events sometimes was difficult not only because of potential disturbance for a large number of people (e.g., due to schedule changes) but also because of presence patterns (i.e., students and researchers working part-time) or tight schedules for doing experiments (no time to shift these activities).

The engagement of the BM/FM/EM is crucial to successful DR. These stakeholders act as gatekeepers, especially related to decisions to participate based on scheduling and comfort. They are responsible for the comfort in the buildings and their assessment affects their choice to opt-in or optout. They are also the ones that assess if a DR event is too much of a disturbance to ongoing core processes at the site. In the hospital environment, the considerations of the EM/FM/BM and control room people is somewhat different in that they always need to be prepared for emergency situations and they always need to be available-which means that participation in a DR event comes second. At the French site, having several part-time working BMs limited their availability and ability to manually participate in DR events.

Although not extensively investigated, it appeared that at all sites there were to some extent already comfort issues-comfort being perceived as falling short-which is likely to affect the willingness of building occupants to accept any further infringements.

The hierarchical lines of communication sometimes worked well to encourage people to actively participate in DR events, but this was not always equally appreciated (e.g., in the UK, having team leaders directly and personally approach employees to participate worked well; like in France when teachers asked their students to participate during class; but in Italy the employees did not like the mandatory shifting of activities during a DR event). In Romania, a peer-to-peer approach, whereby student leaders engaged their fellow students, worked quite well although keeping them engaged and committed was a challenge.

As a general observation, it has become clear that if higher management is not very aware of the potential benefits that DR can bring, or if it is absorbed by other priorities, it is difficult to get them really committed (even when they are sympathetic to DR and the DR BOB project). At the Italian site in particular it was emphasized that a lack of an energy awareness across the organisation and higher management undermines the likelihood of getting commitment from the part of the building occupants.

As for communication to building occupants, information overload and mistakes in communication were mentioned at both the French and Romanian sites as invoking annoyance among building occupants. The Italian site showed how information provision is restricted by organisational policies-e.g., prohibiting the use of social media like Whatsapp during work. Another limiting factor is that not all building occupants are seated behind their computers large part of the day so they need to be informed about DR events differently than by emails only (e.g., face-to-face).

- At all demo-sites there were building occupants who were interested in receiving feedback on the impact of their participation. However, this information was not available. At the French site, the demo-site manager developed his own calculations to be able to provide such feedback, which was considered important to keep these people engaged.

- As for the engagement of the demo-site managers themselves, their role turned out to be crucial at all sites as they fulfilled several roles at all demo-sites:

- Support in the installing of the necessary software and hardware

- Informing and checking regularly with BM, FM and EM-explaining the DR programmes, getting their commitment and support

- Checking notifications and responses 
- Where needed forward notifications and messages around the DR events

- Keeping BM, FM and EM motivated

- Motivating building occupants and keeping them motivated.

When asking the demo-site managers who should take up this intermediary role in future DR $\mathrm{BOB}$ project, they considered the energy manager as the most suitable person to fulfil this role.

The demo-sites managers considered that the engagement of building occupants is important in order to achieve enhanced energy awareness, energy saving and efficiency behaviour-so from an energy efficiency point of view. They concluded that engaging building occupants in actively participating in DR is less relevant for future DR. This is because it took them quite some effort to reach the building occupants and to get them actively engaged, while at the same time the available loads were quite small. For DR in BOBs, a limited number of assets that have large capacity for delivering flexibility (alike industrial DR) is preferred over engaging a multitude of assets and their smaller loads if this means that the diverse users of these assets are to be actively engaged. The exception to this was the Romanian site where the coordinator was quite satisfied with results of the active participation of the students - in terms of peak time savings.

\section{Discussion: Recommendations for Each Readiness Level}

The recommendations provided below (table 3) are generic, and to some extent conditional on:

- The type of scenario: does it ask for active participation of building occupants or not?

- The sort of site: is the BOB a business park, a university campus, a hospital or yet another type of BOB (which may affect organizational and physical integration)

- The country (institutional context-e.g., market for DR)

- Other site-specific circumstances (e.g., related to the types of buildings and infrastructure).

It should also be noted that the recommendations have relevance for DR in BOBs, but they also may be useful for DR in single buildings or building units such as offices.

Table 3. Recommendations for each readiness level.

\begin{tabular}{|c|c|}
\hline $\begin{array}{l}\text { Physical and tech- } \\
\text { nological readiness: }\end{array}$ & $\begin{array}{l}\text { - Check if the machines and other assets are DR-proof and take sufficient } \\
\text { time to make the building systems ready to integrate the DR BOB solution. } \\
\text { - Investigate together with IT services how to integrate the IT infrastructures } \\
\text { and what data limitations may exist. } \\
\text { - Check if older assets allow for remote control. } \\
\text { - Check if the age, (monumental) status and function of the building allows } \\
\text { for participation in a BOB-level DR programme. }\end{array}$ \\
\hline Market readiness: & $\begin{array}{l}\text { - Focus on benefits from power reduction and energy savings in non- or par- } \\
\text { tially developed markets } \\
\text { - Define and calculate a baseline at the beginning of pilot demonstration. }\end{array}$ \\
\hline $\begin{array}{l}\text { Organisational } \\
\text { readiness: }\end{array}$ & $\begin{array}{l}\text { - Create energy awareness from the top to bottom in the BOB } \\
\text { - Focus on the multiple benefits of DR/make your DR solution a strategy for } \\
\text { - Comfonagement to reach their goals } \\
\text { - } \text { and assess if building or asset improvements need to be implemented first } \\
\text { - Assign one single DR contact person for the building users } \\
\text { - Tailor your communication and involve the building users in this process } \\
\text { - Find out what the flexibility profiles and core processes of the building } \\
\text { - } \text { users are and tailor the DR events to match these } \\
\text { - } \text { time } \\
\text { - Beware of cascading impact }\end{array}$ \\
\hline
\end{tabular}




\section{Conclusions}

This paper discussed the demonstration experiences from the H2020 DR BOB project. Over a period of four years (2016-2019) the project developed a Demand Response solution and demonstrated that solution at four pilot sites in blocks-of-buildings. The challenges faced in dealing with the disperse and multifaced nature of BOBs when planning for DR interventions have been identified and structured using the readiness levels' framework.

It was shown that at each demo-site, organisational readiness was a major challenge to the adoption of DR. Clearly, even when the technological and physical requirements for DR are in place and when the market enables both implicit and explicit DR response (e.g., in the UK), successful implementation of a DR BOB solution is not guaranteed unless the organisational challenges have been addressed as well.

The three readiness categories offer the possibility to assess beforehand what is needed for a good match between a DR solution and the specific site-in order to obtain insight in how DR or other energy-related services may be relevant for a specific BOB. As such, we have attempted to provide a tool for those interested in developing propositions offering energy optimization services that are in line with the customer and user needs [3], with the organizational ambitions and priorities at the $\mathrm{BOB}$, the technical and physical starting situation and that offer possibilities to develop value in a functioning market context.

Funding: The work presented was carried out as part of the DR BOB project (01/03/16-31/08/19) which is cofunded by the EU's Horizon 2020 framework programme for research and innovation under grant agreement No. 696114.

Acknowledgments: The authors wish to acknowledge the efforts of the demonstration site coordinators, and the contributions of all those involved in DR-BOB.

\section{References}

1. Crosbie, T.; Short, M.; Dawood, M.; Charlesworth, R. Demand response in blocks of buildings: Opportunities and requirements. Entrep. Sustain. Issues 2017, 4, 271-281.

2. Breukers, S.; Crosbie, T.; Mourik, R.; Heijnen, V. Deliverable: 5.3 Guidelines for Future ProjectsPRELIMINARY; Deliverable for the DR BOB Project; DuneWorks: Eindhoven, The Netherlands, 2019.

3. Breukers, S.; Crosbie, T.; van Summeren, L. Mind the gap when implementing technologies intended to reduce or shift energy consumption in blocks-of-buildings. Energy Environ. 2019, in press. 